\title{
FRANZ BRENTANO: EQUIVOCIDAD DEL SER Y OBJETO INTENCIONAL
}

\author{
Mario Ariel González Porta*
}

\begin{abstract}
This work aims to prove that the brentanian notion of intentionality, and particularly his conceptions of intentional object and immanence, cannot be properly understood but in the boundaries of the author's ontological concerns, particularly his former defense and later denial of the equivocity of being.

RESUMEN Este trabajo se propone probar que el concepto brentaniano de intencionalidad, más propiamente, su teoría de la inmanencia del objeto intencional y el posterior abandono de la misma, no pueden ser comprendidos de modo adecuado sino en el marco de las preocupaciones ontológicas del autor y, en particular, de su defensa inicial y subsiguiente rechazo de la tesis de la equivocidad del ser.
\end{abstract}

\section{Introducción}

El nombre de Brentano ha pasado a la historia de la filosofía en vínculo estrecho con el concepto de intencionalidad tal como este aparece formulado en un célebre pasaje de la PES de 1874. Esto ha llevado a separar esta obra e, incluso, este pasaje, del conjunto de su reflexión. El es, sin embargo, simple momento de una sistemática más englobante, surgiendo de su aislamiento distorsiones inevitables. La correcta comprensión de la teoría de la intencionalidad remite a la de otras teorías del autor, pues se interrelaciona con ellas.

* PUC-SP

KRITERION, Belo Horizonte, $n^{0}$ 105, Jun/2002, p.97-118 
El conjunto de estas, a su vez, experimenta una evolución. En lo que sigue me propongo reintroducir el famoso texto de Brentano sobre intencionalidad en su contexto sistemático y genético-evolutivo para, de este modo, posibilitar una reinterpretación del status ontológico del "objeto intencional".

Tal como Brentano la entendía, no es posible hacer filosofía sino en diálogo con los clásicos, partiendo de su estudio y tomando como referencia a un maestro. En su caso, este maestro es Aristóteles, al cual dedica un concienzudo estudio ${ }^{1}$. Esto significa que, en principio, y salvo indicación expresa en contrario, Brentano considera la doctrina aristotélica como verdadera y solo cuando se presenten dificultades que así lo justifiquen, habrá de modificarla o, eventualmente, rechazarla. La evolución de Brentano está marcada por un partir de Aristóteles, para distanciarse gradualmente, hasta llegar a una ruptura definitiva con el estagirita, ruptura que, al mismo tiempo, señalará su punto de mayor originalidad y madurez reflexiva.

La ontología es un interés constante en Brentano, desde la primera a la última obra ${ }^{2}$. Su disertación tematiza la equivocidad del ser según Aristóteles; su obra posterior vuelve a retomar este tema, focalizándose en la necesidad de reformular la teoría aristotélica de las categorías y, en especial, de la sustancia. Es en el contexto de esta preocupación ontológica que debemos situar el concepto de intencionalidad y sus alteraciones. Más concretamente, el status del objeto intencional debe ser entendido en el marco de la evolución brentaniana con respecto a la temática de la equivocidad del ser ${ }^{3}$.

\section{2. «Sobre la equivocidad del Ser según Aristóteles»}

En su tesis doctoral Brentano parte de un conocido pasaje de la "Metafisica", en donde Aristóteles, presuponiendo que cada ciencia debe tener un objeto y que la metafísica ha de ser ciencia, llama la atención sobre el hecho de que "ser" se diga en una multiplicidad de sentidos. El filósofo griego procede entonces a diferenciar estos sentidos, estudiando su vínculo con el objeto de la ciencia en cuestión. Ahora bien, la metafísica es la ciencia del ser en cuanto ser. Se debe entonces establecer cuáles son los modos propios e impropios de este "ser" para fijar la unidad del objeto de la disciplina que nos ocupa.

1 Las das primeras manografías de Brentano san ejercicios académicas de interpretación del texto aristotélico. Haber colocado la reflexión brentaniana en el marco de sus verdaderas coordenadas es mérito innegable del imprescindible libro de Smith, Barry: Austrian Philosophy. The legacy of Franz Brentano. Chicago y La Salle (Tllinois), 1994.

3 Así camo la equivocidad del ser repercute sobre la idea de intencionalidad, esta, aún cuando en menor medida, también lo hace sobre aquella, doligandb a reformular el problema y a distinguir allí donde antes no se lo hacía. 
Las varios sentidos del ser distinguidos en el texto aristotélico se dejan reducir, según Brentano, a cuatro ${ }^{4}$ :

1. el ser por accidente,

2. el ser verdadero,

3. el ser en cuanto potencia o acto y

4. el ser en cuanto sustancia o modificación de la sustancia (o ser en el sentido de las categorías).

Algunas rápidas observaciones a esta división:

1. Los dos primeros sentidos diferenciados son impropios; los dos últimos, propios.

2. El ser por accidente es desconsiderado como objeto de la metafísica y, más aún, de toda ciencia (VMBSA,39).

3. También el ser verdadero será excluido como objeto de la metafísica, aun cuando, a diferencia del caso anterior, será posible una ciencia del mismo, la «lógica» (VMBSA,39).

4. El ser en el sentido de la potencia y el acto habrá de ser tratado en íntima relación con el ser en el sentido de la sustancia y las categorías, ya que ambos constituyen el objeto de la ciencia primera.

5. La importancia, no obstante, del ser en el sentido de las categorías, se revelará infinitamente superior, mereciendo, por tal motivo, un estudio minucioso del cual resultarán, a su vez, una nueva multiplicidad de sentidos.

6. Del ser por accidente y del ser en cuanto potencia o acto poco se nos dice y menos aún de interés. Nos tenemos que concentrar, pues, en los otros dos sentidos dado que, como veremos, será con respecto a su relación que habrá de evolucionar el pensamiento de Brentano. La tesis básica reza: no todo lo que es, es «real» o: existen, «son», en sentido propio, cosas que, no obstante, no son «reales».

¿Qué es el ser en el sentido del «ser verdadero»?

1. "Ser" y "es", nos dice Brentano siguiendo a Aristóteles, también significan que una cosa es verdadera; no ser, que ella es falsa y, esto, tanto en la afirmación cuanto en la negación. Así, por ejemplo, decimos que Sócrates es músico, porque ello es verdadero o bien que Sócrates no es blanco, porque también ello es verdadero. Decimos, en cambio, que el diámetro no es conmensurable, porque es falso que lo sea.

2. Verdad es el acuerdo entre el pensamiento y la realidad (VMBSA,28ss.)

4 Dejo de lado, ahora y en lo que sigue, la pregunta de si la interpretación brentaniana de Aristóteles es sustentable o no. 
3. y, en última instancia, una propiedad de juicios.

4. Dado que juicios son actos del espíritu, el ser verdadero se sitúa en el alma. La verdad "está" en ella.

5. Del hecho de que la verdad se encuentre "en" el alma pasa Brentano a afirmar, por medio de una problemática inversión, que, entonces, todo lo que «está en" el alma participa del modo de ser propio del ser verdadero. Como existencia "mental", opuesta a toda existencia "real", el ser verdadero abarca todo aquello que meramente existe "en" el alma y no en la realidad (externa). La oposición básica es, por tanto, entre lo que es «en» la conciencia y lo que es «fuera» de ella, siendo que lo que es «en» la conciencia, tiene un ser disminuido, aun cuando, de alguna forma, posea algún tipo de «realidad» (Realität).

6. Se ha observado en repetidas ocasiones que el concepto brentaniano de intencionalidad aparece por primera vez en el trabajo sobre la psicología de Aristóteles (1867). Ahora, es interesante desde el punto de vista sistemático y no una mera curiosidad histórica - que sus orígenes ya se constaten en la tesis doctoral (1862), algo que ha sido pasado por alto por los comentadores hasta el momento. Ya aquí, sin embargo, Brentano expresa claramente la idea intencional y, esto, con el mismo vocabulario escolástico del «ser objetivo" con el cual habrá de formularla en 1867 (PA,79ss.) y 1874 (PES,I,124-125)5. El ser «objetivo», por tanto, era pensado por Brentano, en un primer momento, en el marco del ser verdadero y como caso particular del mismo.

7. Si la idea de intencionalidad está claramente presente en 1862, esto no significa, no obstante, que sus implicaciones teóricas hayan sido desarrolladas y que tengamos una posición idéntica a la de 1874. Se nos dice que todo lo que está «en» el alma tiene algún tipo de «realidad» (Realität) (VMBSA,37). No obstante, ¿qué significa esto? La verdad es propiedad de juicios y juicios son actos. Pero, ciertamente, no todo lo que está «en» el alma es un acto y, por lo tanto, se engloba aquí lo que, desde un punto de vista posterior, será lo intencional y lo no-intencional. Si introducimos esta diferencia, entonces no resulta claro qué significa propiamente el "ser verdadero" $\mathrm{y}$, menos aún, qué significa el que a todas sus formas corresponda el mismo tipo de ser. Visto desde el punto de vista de 1874, el ser verdadero parece comprender en 1862 dos cosas diferentes, el acto y el objeto intencional. Si, como es sabido, posteriormente el acto será considerado real, en tanto que el objeto intencional no, en la tesis doctoral se nos habla indistintamente de ambos.

5 "Sogar das Nichseiende ist, weil es ein Nichtseiendes ist, in dieser Weise ein Nichtseiendes seiend, also ein

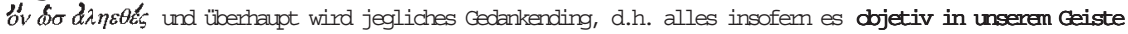
existienend, Subject einer wahren affirmativen Behauptung werden kann, dazu gehören. Nichts was wir in un

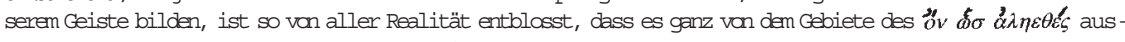
gechlossen wäre.» (VMBSA, 37. Negritas mías). 
8. Dado que no cabe duda de que el alma es una sustancia y que el ser mental es ser «en» la mente, ¿en qué sentido el «es» en ella? Esto nos conduce a un nuevo punto.

Distinguir entre los varios sentidos del ser, delimitar los que son «propios» de los «impropios», tiene como resultado esencial el oponer la existencia puramente mental a la real, el ser "en" la mente al ser "en" la realidad (más precisamente, en la realidad "externa"), fijando de este modo el objeto de la metafísica, la cual habrá de referirse únicamente a este último (VMBS,40). Esta idea encuentra amplia difusión a mediados del siglo XIX. No-metafísico es cualquier punto de vista que se mantenga inmanente a los datos de la conciencia. Brentano parece seguir esta tendencia sin problematizarla. La metafísica se ocupa del ser «fuera» del espíritu, la lógica del ser «en» el espíritu; aquella estudia lo que verdaderamente es, esta el ser men$\mathrm{tal}^{6}$. La referencia prioritaria al problema del objeto de la metafísica pasa a ser la fuente del carácter insatisfactorio de la elaboración del concepto de ser verdadero y, por ello, es de esperarse algún avance cuando la una se independice de la otra ${ }^{7}$.

\section{3. «Psicología del punto de vista empírico»}

La PES marca una evolución cualitativa en el pensamiento de Brentano, quien del comentario de Aristóteles pasa ahora al trabajo sistemático. El concepto de intencionalidad es formulado "explícitamente" por primera vez en esta obra. Como se sabe, característico de esta formulación es que:

1. "Intencional" aparece como propiedad de ciertos objetos y no de "actos" o de la relación de la conciencia a algo.

2. El objeto intencional es «inmanente» a la conciencia. «Intencionalidad» es un atributo de los objetos «de» la conciencia y no una relación de la conciencia a una entidad exterior a ella (PES,I,124-125).

Pero hay algo más.

La noción de «objeto intencional» en PES, como ya antes lo hacía su equivalente, la de "ser objetivo", es pensada por Brentano en el marco de una

6 Igualar la diferencia "ser sustancia - ser verdadero" con la de «fuera del espíritu - en el espírituw produce dificultades y es, en el mejor de los casos, cuestionable. La distinción aristotélica de los sentidos del ser parece ser superpuesta sin más al dualismo modemo entre mundo intemo y extemo.

7 Sólo lo que es fuera del espíritu, existe verdaderamente. ¿No hay pues nada «en» el espíritu que sea verdaderamente existente? ¿Y la propia alma, no es ella una sustancia? ¿Y sus actos, no son reales? Después de 1874 la conciencia y sus actos son verdaderamente existentes y una metafisica, como lo muestra el ejemplo del propio Brentano, tiene en ellos un abjeto esencial. 
concepción general del ser caracterizada por la tesis de la equivocidad. Ella supone la diferencia entre el «ser» y el «ser real»o, más concretamente,

1. que «ser» y «ser real» no son la misma cosa;

2. que no todo lo que "es", es real;

3. que hay cosas que "son" y, no obstante, no son reales;

4. o que hay "Irrealia"8.

En definitiva, el objeto intencional "es", pero no es "real", ni en la conciencia, ni fuera de ella. El no es real "en" la conciencia pues lo único «real» es el acto y la única forma en que puede estar dado en éste es justamente como objeto, o sea, como correlato intencional (PES,I,124-125).

Con lo dicho anticipamos y resolvemos insuficiencias presentes en las lecturas propuestas por la crítica hasta el momento:

1. El objeto intencional es «inmanente». De eso no cabe duda. El problema radica en precisar en qué consiste su inmanencia. Irreflexivamente, tendemos a pensar la oposición entre inmanencia y transcendencia como entre un dentro y un fuera espacial ${ }^{9}$. Así, o algo es real «en» la conciencia o algo es real «fuera» de ella; o, si algo no es real «fuera» de la conciencia, entonces es real «en» ella. Pues bien, para pensar correctamente la inmanencia brentaniana es esencial percibir que la oposición entre un ser real «en» la conciencia y un ser real «fuera» de la conciencia, no es exahustiva y establece una disyunción donde no la hay. La razón es la siguiente: la tesis de la equivocidad obliga a reformular los conceptos de inmanencia y transcendencia. El hecho de que el objeto intencional, en cuanto inmanente, no sea real «fuera» de la conciencia, no quiere decir que, entonces, él sea real «en» la conciencia.

2. El error al cual apuntamos se puede presentar de otra manera. Si, en vez de entender inmanencia como ser real "en" la conciencia, se la piensa simplemente en base a la noción de ser, queda aún, por lo menos, una indeterminación. Se nos dice que el objeto intencional no «es» en la conciencia y, asimismo, sin solución de continuidad, no obstante, que el "es en" ella. Ahora, ¿no es esto contradictorio? Ciertamente lo es si, como acontece usualmente, no se nos proporciona ninguna ulterior aclaración; no lo es, si se tiene en cuenta que, ese su ser en la conciencia, no es, en realidad, otra cosa que su noser real. El objeto intencional no «es real" en la conciencia, pero "es" en ella $\mathrm{y}$, en consecuencia, en este sentido, es inmanente.

8 Como veremos, la repercusión de ésta tesis no se restringe al problema del abjeto intencional. El no es el único tipo de cosas que tiene, de algún modo, ser, aun cuando no sea real.

9 Obsérvese que no es sobre el hecho del carácter espacial de la metáfora, sobre lo que llamamos la atención ahora. 
3. Reuniendo las dos líneas expuestas digamos: el ser «en» y el ser «fuera» no son necesariamente el mismo ser que simplemente se "traslada" de un lugar a otro; ese "traslado" implica en realidad un decisivo cambio en su «tipo». El problema básico, incluso de la propia inmanencia, no es «donde» el objeto intencional es, sino «cómo» ${ }^{10}$. Este último punto merece aún algunas consideraciones.

La idea brentaniana de intencionalidad está comprometida de modo esencial con una teoría de la percepción. En efecto, siendo la representación sensible (sinnliche Vorstellung) - sea imaginación, sea percepción — la forma básica de intencionalidad, es claro que el status del objeto intencional está indisolublemente vinculado al del objeto perceptivo. Si esto es así, entonces el concepto de intencionalidad brentaniano supone una tesis cuyo sustento no es "fenomenológico", a saber, la «idealidad» de las cualidades secundarias. No hay posible argumentación en primera persona para la misma, sino solo en tercera ${ }^{11}$. Esencial para entender el concepto brentaniano de intencionalidad es ver en el mismo la confluencia de dos tradiciones: Aristóteles y la modernidad o, la teoría hilemórfica y el distingo entre cualidades primarias y secundarias. La cuestión es cómo deben ser pensados cada uno de estos momentos para que se integren sin contradicción. Si el concepto de intencionalidad se remonta a Aristóteles, lo hace en el marco de su teoría de la percepción. Ahora, el punto básico es que, en la concepción aristotélica, la "forma" puede estar presente de dos maneras: justamente, en cuanto lo que define al «sentido» es la capacidad de recibir formas sin materia, yo puedo decir que la forma «está» en el objeto y que «está» en mí en cuanto percibida y, eventualmente, que es «la misma» forma. Todo esto ya no puede decirse cuando se afirma la subjetividad de las cualidades secundarias. El hecho fundamental es que Brentano no puede tomar la teoría de las cualidades secundarias y simplemente ligarla a otra sino que, al hacerlo, da a la misma un giro particular. Las cualidades secundarias no son meramente localizadas "en" la conciencia sino que, al mismo tiempo, reciben un status similar al que antes poseían las formas en Aristóteles. Es ese status que intentamos calificar como ser «objetivo» o «intencional». En la filosofía moderna cualidades secundarias «son» en nosotros; en Brentano, solo son en nosotros de modo objetivo. En una

10 En un texto extremadamente esclarecedor Brentano define la inmanencia como sinónimo de ser intencional. No es que el ser objetivo fuese «inmanente», como si tuviese una propiedad más, sino que ser inmanente no es otra cosa que ser "objeto" de la conciencia (WE, 87.ss.). La diferencia entre el abjeto irmanente y el transcendente no es la diferencia entre dos tipos de dbjetos, sino entre dos sentidos del término "dojeto". 11 Este no es el único punto de incoherencia en el análisis "descriptivo" (fenomenológico) de Brentano. 
visión ingenua podríamos decir que el color no está «en» el objeto sino en el alma. Ahora, este «donde» del color en realidad encierra el problema fundamental del «cómo». ¿En qué sentido el color está «en»el alma? No se trata meramente de que el color se desplace desde "fuera" para "dentro" sino que, al mismo tiempo, cambia su modo de ser. El objeto es blanco, el sujeto que percibe el blanco no es él mismo blanco (PA,79ss; PD,26). Ser-blanco es una propiedad de la cosa, no de la conciencia que la menta. El objeto no está en la percepción de otro modo que no sea el intencional; de ahí que en el «existeen» (Inexistenz) (PES,I,125), el "en" no indica propiamente un lugar, sino una forma de existencia.

El problema de la equivocidad del ser no está presente en PES únicamente en cuanto presupuesto sistemático del concepto de objeto intencional, sino en claros y explícitos pasajes referidos de modo directo a la propia definición de intencionalidad. Es sobre su base que Brentano observa que, en la teoría de la existencia mental de Philo, la inexistencia es confundida con la existencia en sentido propio, el ser mental con el real, con lo que se llega a una teoría de las ideas y del Logos contradictoria (PES,I,125). Algo similar vale, según nuestro autor, para San Agustín y para San Anselmo (PES,I,126; Compare WE,117).

Finalmente, la tesis de la equivocidad del ser constituye la base implícita de la teoría de todos y partes, con la cual Brentano termina el primer volumen de la PES lo cual, no siendo obvio en el texto de 1874, habrá de quedar retrospectivamente en claro a partir de 1904. La PES se propone analizar la conciencia en sus elementos con el objetivo de establecer sus partes simples y los modos en que estas se combinan a los efectos de la composición del todo. Por tal motivo, para hacer efectivo su análisis de la conciencia, Brentano precisa de una teoría general de todos y partes. Esta se guiará por el principio, retomado de Aristóteles, de que es imposible que al mismo tiempo algo sea una "cosa real" (reelles Ding) y una multiplicidad de cosas reales (relle Dinge) o, explicitando lo dicho:

1. Si las partes son reales, el todo no lo puede ser e, inversamente, si el todo es real, las partes no lo son.

2. Una cosa (Ding) no puede ser parte (Teil) de otra cosa e, inversamente, las partes de una cosa (die Teile eines Dinges) no pueden ser jamás cosas.

Subyacente a esas tesis se encuentra, en última instancia, la idea de que todos y partes (o cosas y partes) no pueden "ser" en el mismo sentido.

Para simplificar la exposición de lo que sigue, comenzemos por algunas definiciones. Denominaremos «colectivo» (Kollektive) a una reunión o tota- 
lidad de cosas (Dinge). El «colectivo», justamente por componerse de cosas, no es jamás una cosa. Pero, si una cosa no puede componerse de cosas, sí puede componerse de partes (Teile), esto es, ella puede contener una multiplicidad sin contradecir su unidad. Llamaremos "divisivos" (Divisive) a las partes de una cosa que, a su vez, no son cosas.

Establecidas las definiciones nominales, se trata ahora de esclarecer el status ontológico del Divisivo. Dado que el mismo es diferenciable de la cosa aun cuando no subsiste sin ella, podría pensarse que el distingo entre ambos es meramente mental o que se trata de una pura abstracción. Sin embargo, tal lectura dista de solucionar todos los problemas, sobre todo porque Brentano también afirma las tres tesis siguientes:

1. El divisivo no puede existir sin la cosa; esta, no obstante, puede existir sin él.

2. Divisivos son realmente separables de la cosa, siendo posible que dejen de existir sin afectarla.

3. Divisivos son realmente separables entre sí, esto es, pueden dejar de existir, sin efectar a otro divisivo ${ }^{12}$.

Las tesis 2 y 3 evidencian que un divisivo es algo más que una mera abstracción, pues, si este no fuera el caso, ¿cómo podrían ellos ser realmente separados de la cosa y entre sí? Ahora bien, ¿qué es ese algo más? Veremos que este es un punto central en el análisis de la conciencia.

Existe la unidad de la conciencia. La totalidad del alma en un instante dado $^{13}$ no es una sumatoria o agregado de cosas, sino una cosa única. Todos los fenómenos psíquicos que se encuentran en nosotros simultáneamente pertenecen a una realidad (Realität) unitaria, esto es, en tanto fenómenos parciales, ellos constituyen un fenómeno psíquico, del cual sus partes no son cosas diferentes o partes de cosas diferentes (PES,I,323). Lo dicho implica:

1. La unidad de la conciencia ni es sinónimo de simplicidad ni excluye lo múltiple. El alma tiene unidad, pero no es simple (PES,I,11). En cada momento de la conciencia hay multiplicidad, aun cuando, ni en sus más complejos estados, ella pierde su unidad. La simultánea unidad y complejidad de la conciencia se manifiesta en tres momentos:

a) la conciencia es un acto que siempre implica otro, la co-conciencia de sí mismo;

\footnotetext{
2 Aun cuando, en principio, también puede haber divisivos que solo existen con otros divisivos o presupongan otros divisivas.

13 Obsérvese que, curiosamente, Brentano no considera la perspectiva temporal en el análisis de la unidad de la conciencia.
} 
b) varios actos pueden dirigirse al mismo objeto (por ejemplo representar y amar); char).

c) actos con objetos diferentes pueden coexistir (por ejemplo ver y escu-

2. La unidad de la conciencia no significa homogeneidad (Gleichteiligkeit) (PES,I,237).

3. La unidad de la conciencia no significa indivisibilidad (Unteilbarkeit) (PES,I,236,243).

El punto realmente problemático no es el que Brentano coloca en primer plano, es decir, la oposición entre unidad y multiplicidad, sino el hecho de que él vaya más allá para afirmar la separabilidad real de los momentos de la multiplicidad, tanto entre sí (PES,I,234-235), como respecto de la unidad (PES,I,224).Veamos esto en detalle.

Entre los actos hay dos relaciones posibles, la de separabilidad recíproca y unilateral. Así, por ejemplo, es posible separar realmente el acto de representar del de juzgar, pero no de modo recíproco, pues el primero supone el segundo ("partes unilateralmente divisivas": einseitig divisive Teile); por el contrario, se puede separar real y recíprocamente el acto de ver y el de oír ("partes recíprocamente divisivas": gegenseitige divisive Teil). Todo esto, sin embargo, no pasa de un mero postulado. No cabe duda, ex-hipotesis, que los actos psíquicos, en un momento dado, pueden ser complejos y componerse de varios actos; sin embargo, ¿qué nos prueba, que estemos frente a una unidad? Los argumentos brentanianos pretenden ser esencialmente fenomenológicos:

1. En el primer caso, es necesario que los dos actos tengan el mismo objeto, pues si no lo tuvieran, no serían lo que son. El afecto supone la representación. Ahora bien, no es posible, tener afecto por una cosa y representarse otra diferente de esta, justamente porque el afecto, al suponer la representación, supone también la identidad del objeto de ambos (PES,I,225-226).

2. Puedo establecer un nexo entre diferentes actos, por ejemplo ver y oír, los puedo comparar y diferenciar; esto, no obstante, no sería posible si, de alguna forma, ellos no conformasen una unidad (PES,I,226).

Obsérvese que Brentano tiene que descartar dos cosas al mismo tiempo: cuando un mismo objeto es objeto de dos actos (como representar y amar), estos no son una reunión de varias cosas y, no obstante, no siéndolo, son separables, tal que el uno puede seguir existiendo en su identidad sin el otro ${ }^{14}$.

14 El hecho de que los actos puedan ser realmente separados unos de otros parece ser un buen argumento para indicar que son meramente "colectivos", esto es, reuniones de cosas. 
Pero, ¿qué significa entonces “su identidad”? Aparentemente, representar el «mismo» objeto. Es lógico, por otra parte, que para poder comparar dos fenómenos, los tenga que integrar en una conciencia única. Eso no dice absolutamente nada, sin embargo, respecto a si ellos constituyen una unidad real o no. La teoría brentaniana, con su tendencia a igualar el saber de la unidad con la unidad de una "cosa", torna confuso el propio concepto de unidad de la conciencia, superponiendo los planos fenomenológico y ontológico.

\section{4. «Sobre el concepto de verdad»}

Conforme con la orientación general de seguir a Aristóteles ya indicada, Brentano retomaba sin comentario en su primera obra la tesis de la verdad como adecuación. A partir de 1889 se produce su primer distanciamiento con respecto al estagirita, el cual sucede justamente en este punto (WE,3ss.). Brentano sigue concordando con su maestro en que la verdad es el acuerdo entre pensamiento y ser; haya necesario, no obstante, para explicar la posibilidad de juicios existenciales negativos verdaderos ${ }^{15}$, diferenciar la cosa del «ser» de la cosa. Reformula entonces la teoría de la correspondencia, situándola no entre el pensamiento y la cosa, sino entre el pensamiento y el «ser de la cosa», en el caso de la proposición afirmativa verdadera, y el «no-ser de la cosa», en el caso de la proposición negativa verdadera ${ }^{16}$.

Ya hemos observado que en 1862 Brentano sigue a Aristóteles en la tesis de que verdad y falsedad son propiedades del acto de juzgar. Si tenemos en cuenta este hecho, se imponen algunas consideraciones con respecto a la teoria brentaniana del juicio de 1874 y las innovaciones que experimenta en 1889. La primera es que estamos frente a una teoría del juicio, no de la proposición y, más aún, frente a una teoria del juicio que prescinde absolutamente de «estructuras proposicionales». Esta es una constante en Brentano. Según la doctrina de PES, en el juicio se afirma o niega el contenido de una "representación" (Vorstellung) y esta no es semánticamente articulada. La introducción del «ser de la cosa» (Sein des Dinges) ${ }^{17}$ no conlleva a alteraciones en este punto. La única diferencia es que si antes el juicio se dirigía a la cosa

15 O sea, de juicios verdaderos en los cuales se niega la existencia de algo cuya verdad, por tanto, no puede consistir en el acuerdb entre el juicio y la cosa, ya que justamente esta cosa no existe.

16 Existen otras modificaciones importantes aunque no son de relevancia para nuestro tema y, por tal motivo, no las analizamos.

17 En la discusión posterior dentro de la escuela, el "ser de la casa" es igualado a menudo a la noción de "estados de cosas" (Sacvherhalt), que fuera introducida en la discusión por Stumpf y tiene claras diferencias con la brentaniana. Por tanto, aplicada en este momento, la expresión referida es anacrónica. Camo, no dostante, existe un continuo de discusión entre la primitiva idea brentaniana del "ser de la cosa" y Stumpf (o Marty), se puede mantener por comodidad la misma como, por otra parte, no es inusual entre los críticos. 
representada, ahora lo hace al "ser de la cosa". Ahora bien, ¿qué es este "ser de la cosa" (Sein des Dinges) diferente de la "cosa" (Ding)? Desde un punto de vista lingüístico contemporáneo el mismo es simplemente la forma sustantivada de la estructura "que... es" ("dass p"); para Brentano, no obstante, se trata de un objeto particular (y simple) que funda la verdad del juicio: el «ser del caballo» es lo que hace verdadero el juicio: «el caballo es». Otra importante diferencia entre la concepción brentaniana y la actual es que la función del "ser de la cosa" es exclusivamente veritativa y no semántica: es aquello que funda el valor de verdad del juicio y no aquello que fija el sentido del enunciado. Así como no hay estructuras proposicionales en el horizonte del pensamiento brentaniano, tampoco está presente un modo de consideración propiamente semántico. El problema de la significación simplemente no aparece $^{18}$.

Dado que el "ser de la cosa" habrá de ser asimilado a la «existencia»y «la cosa» a la «realidad», la reformulación de la teoría de verdad en Aristóteles conduce a retomar el problema de la equivocidad del ser (WE,28). Veamos esto en detalle.

a) Ya sabemos que en el escrito sobre el carácter equívoco de «ser» había una cierta desproporcionalidad entre la atención que Brentano dedicaba, por un lado, al ser accidental y al ser en cuanto potencia y acto y, por otro, al ser verdadero y al ser en el sentido de la sustancia.

b) En el desarrollo ulterior estos dos sentidos privilegiados pasan a ser

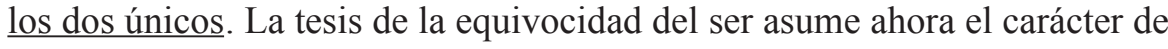
una dualidad entre el «ser verdadero» y el «ser en el sentido de la sustancia». Con esta concentración viene un cambio del vocabulario: la oposición deja de formularse preferentemente en términos de ser-verdadero - ser-sustancial para pasar a hacerlo en términos de «existir» y «ser real» o "existencia" y "realidad". La diferencia básica que ha de ser mantenida es: no todo lo que existe, es real.

c) En la misma medida en que la distinción de sentidos del ser se reduce a dos únicos, ella se independiza del problema de la unidad de la metafísica como ciencia para pasar a ser un fin en sí misma, eventual punto de partida de otras delimitaciones de relevancia.

d) En un fragmento de 1902, esta línea de pensamiento parece llegar a su culminación (WE, 32): ya no meramente se distingue el «existir» del «ser real», sino que se introduce una subordinación entre ambos que, por otra

18 Si no hay propiamente semántica en Brentano, sí hay pragmática. El sentido que se considera no es el del enunciado, sino propiamente el de la enunciación. 
parte, invierte la presente en VMBSA. El "ser verdadero" es ahora el sentido primario que incluye, como su caso particular, el ser en cuanto real. Todo lo que es, lo es, se nos dice, por lo menos "también" en el sentido del ser verdadero, aun cuando, claro está, no todo lo que es en el sentido del ser verdadero, es propiamente real.

¿Por qué la noción de "existir" sustituye la de «ser verdadero» como uno de los términos de la oposición? Quizás estén en juego dos razones básicas. Sin duda que de aquello que se dice verdad, en algún sentido se predica existencia. Además, el ser verdadero sigue siendo propiedad del juicio y, en tal medida, algo "psíquico», pero lo que hace verdadero al juicio (el ser de la cosa), no obstante, parece no ser algo "psíquico". Brentano dice muy poco sobre el "ser de la cosa", constituyendo la cuestión de su verdadero status un difícil problema. Es plausible que su postulado sea el momento de mayor «realismo» en el desarrollo del pensamiento brentaniano. Ahora, aún con más motivo que en 1862, hubiera sido necesario un análisis de los varios significados del "ser verdadero" 19 . Brentano, sin embargo, no lo ofrece y, en ningún momento, observa que el "existir" puede incluir dos cosas en principio diferenciables: el existir como algún tipo de ser «ideal» y, dado que la verdad solo se encuentra en el entendimiento, el existir en el sentido de todo aquello que, sin ser realmente, es al menos en el entendimiento. Lo único que Brentano ofrece es una caracterización negativa y debemos conformarnos con ella.

\section{5. «Psicología descriptiva»}

En la PD el análisis "fenomenológico", que era en PES propedéutica a una psicología naturalista, gana autonomía y se transforma en disciplina con valor propio. Por otra parte, al mismo tiempo que la psicología empírica deviene «psicología descriptiva», se consolida el programa de una fundamentación de la filosofía en la misma. Para nuestro tema importa recalcar la existencia de dos puntos de continuidad entre 1874 (PES) y 1891 (PD):

1. En 1891 se sigue afirmando el principio de que la unidad del alma no implica su simplicidad (PD,10).

2. Asimismo, la explicitación del nexo entre unidad y multiplicidad conduce, como en PES, a que el análisis del alma se concentre en el establecimiento de las relaciones entre las partes y el todo $(\mathrm{PD}, 10)$.

19 O, formulada la cuestión de otro modo, de aquello a lo cual en cada caso se contrapone el ser real, sea el ser verdadero (1862), sea el ser existente (1889). En 1862 esta ausencia amenazaba con nivelar acto y dojeto, ahora, dojeto y ser de la casa. 
Indicadas las constancias, la pregunta es por las alteraciones. Estas conciernen, principalmente, a la extensión y resultados de la teoría de todos y partes, la cual ahora se presenta completa y no ya como un mero fragmento.

1. Las partes de las cuales se trataba en PES eran únicamente de un tipo, a saber, realmente separables. En PD permanece inmodificado lo que respecta a este tipo de partes, mas se agrega una teoría exahustiva de partes que son distinguibles, pero no separables (PD,12ss. $)^{20}$. Allí donde solo hay partes simples desde el punto de vista de la separabilidad real, hay aún partes desde el punto de vista de la diferenciabilidad. Llamaremos a las mismas "partes (meramente) distinguibles" (distinktionelle Teile).

2. Existen cinco tipos de partes (meramente) distinguibles. Ellas se subdividen en dos grupos: las distinguibles en sentido propio e impropio. En sentido propio tenemos a su vez cuatro tipos:

a. partes "interpenetradas" o de "inseparabilidad distincional recíproca" (sich durchwohnende Teile), por ejemplo cualidad, claridad y posición del objeto espacial;

b. partes lógicas o inseparables distincionalmente de modo unilateral como, por ejemplo, la relación del sentir, el ver y el ver el rojo;

c. partes del correlato intencional (es claro que no puede haber un hombre pensado sin un hombre pensante ${ }^{21}$ ) y, finalmente,

d. partes de la conciencia, primaria y secundaria.

En otro grupo de partes distinguibles tenemos aquellas que lo son en un sentido «modificado» (modifizierende Teile), por ejemplo la diferencia entre «x está frío», que es genuinamente distincional, $\mathrm{y}$ "x siente frío», que es distincional sólo en sentido modificado.

3. Mientras que las partes de la conciencia en PES eran básicamente los actos y de lo que se trataba era de la relación entre los mismos, en PD se adiciona tanto la perspectiva de un análisis detallado del objeto y sus momentos como, principalmente, de la relación de este con el acto. Lo único que se decía al respecto en PES era que el mismo objeto puede participar de actos diferentes.

4. Ya en 1874 la expresión "contenido" (Inhalt) aparece en la definición de intencionalidad; es ahora, no obstante, en 1891, que se consolida como

20 Una razón más para no considerar las partes de 1874 como meras abstracciones.

21 Brentano insiste, con razón, en la estricta correlatividad de lo pensado y el acto de pensarlo: es dbvio que no puede haber un hombre pensado, sin un hombre pensante. Sin embargo, nada nos dice aquí al respecto de la cuestión inversa, que es esencialmente más compleja y sistemáticamente más relevante, o sea, por una parte, si puede haber un hombre pensante sin uno pensado y, por otra, sobre todo, en qué sentido el hombre pensado es correlato del pensante. El tema es abordado en esta perspectiva en el difícil texto de la carta a Marty del 17 de marzo de 1905 (WE, 87-89). 
«término técnico», al cual se caracteriza positiva y detenidamente. El tiende a sustituir las otras expresiones que, anteriormente, eran tratadas como sinónimos tales como, por ejemplo, objeto (Gegenstand, Objekt). No obstante, si hay una cierta novedad en la precisión del vocabulario, no la hay desde el punto de vista propiamente conceptual. Los contenidos no son otra cosa que el correlato de la intención, que existe, pero no es real. El vínculo intencional tiene lugar entre algo real y algo no real (PD,21-22) 22 .

5. Coherente con lo anterior, la teoría de la percepción reafirma el hecho de que la relación intencional básica es la perceptiva, que el objeto de esta no es real y que es «inmanente» (PD,21-22).

6. Ahora bien, no solo el objeto, sino conjuntamente con él, la propia relación del acto intencional al mismo queda ubicada como algo extremadamente especial, no reductible a cualquier otra y merecedora de una categoría propia (PD,21-22). De modo explícito ella es considerada como diferente de una relación entre dos actos. Esta última puede ser de real separabilidad (unilateral o correlativa), mientras que, en el primer caso, no hay separación real. El objeto y el acto constituyen una realidad en la cual sólo se pueden distinguir dos momentos. Más aún, la posibilidad de distinguir entre ambos es de un tipo especial, diferente de la que existe en el caso de la "interpenetración recíproca" (sichdurchwohnende Teile) o de las "partes lógicas" (logische Teile).

Una última observación con respecto a $\mathrm{PD}$, cuya relevancia habrá de evidenciarse en lo que sigue. Si nos atenemos a esta obra el «wirken», es decir, la capacidad de ejercer y padecer acciones causales, sería elemento definitorio del real (para lo cual habría en alemán, ciertamente, un buen sustento lingüístico). No obstante tenemos que ser cuidadosos porque, según otros textos explícitos de Brentano contra Marty, no se debe confundir real y causal (WE,108).

6. El reismo: «EI distanciamiento-abandono de los irreales» ("Die Abkehr vom Nichtrealen") y «La teoría de las categorías» ("Kategorienlehre")

A partir de 1904 comienza a operarse un cambio radical en el pensamiento de Brentano, que se prolongará sin interrupción hasta su muerte: se trata de la opción radical por el "reismo" (Reismus). La tesis defendida es que no existen «Irreales» (Irrealia, Nichtreales), que todo lo que es, es real. Obvia-

22 En 1891 «intencional» ya no es más la propiedad de un abjeto, sino de una relación en la cual la conciencia se encuentra $(\mathrm{PD}, 21)$. 
mente que, para entender lo que Brentano niega ahora con tanto énfasis, es esencial saber qué afirmaba hasta ese momento. La confirmación de la tesis del presente trabajo es en buena medida retrospectiva, esto es, los últimos textos de Brentano que, de forma explícita, procuran refutar la posición que ha sido sostenida hasta este momento, ayudan a comprenderla mejor. La crítica de los propios presupuestos es, al mismo tiempo, una crítica de Aristóteles y una ruptura definitiva con él.

¿Qué son «irreales» (Irrealia)? La teoría de los irreales abarca cosas a primera vista extremamente heterogéneas, reunidas al azar y sin ningún criterio, entre las cuales es difícil encontrar un núcleo común. Dentro de este grupo genérico reciben un tratamiento especial y evolucionan de modo asimétrico los siguientes items: 1. Contenidos (Inhalten), 2. estados de cosas (Sachverhalte o Sein des Dinges), 3. colectivos, 4. partes de un continuo, 5. el infinito, 6. espacio y tiempo, 7. las relaciones, 8. el vínculo sustancia-accidente y 9 . todos y partes ${ }^{23}$.

El concepto brentaniano de «irreal» posee una unidad puramente negativa, la cual solo tiene sentido y puede fijarse si retornamos a la doctrina de la equivocidad del ser (AN,347ss.; K,4ss.). «Irreal» es todo aquello que es (existe), pero no es real. La teoría de 1904 se deja resumir en las siguientes tesis:

a. Lo único que "es", es real (reell) (no hay algo que sea y no sea real).

b. Ser real, es ser una "cosa" (Ding, Wesenhaftes, $\left.\operatorname{Res}^{24}\right)$.

c. Todo lo que es, es una cosa.

d. No todo lo que es real, existe ${ }^{25}$.

Vinculado a las tesis anteriores, pero no sin más idéntico a ellas, se encuentra el siguiente principio - negación directa del axioma aristotélico que era punto de partida en 1874 - : un todo real se puede componer de partes reales; de lo cual se sigue, que una cosa puede ser parte de otra cosa o que las partes de una cosa pueden ser a su vez cosas.

La superación de los irreales no meramente implica que solo el ser real "es" sino, asimismo, que solo él puede ser representado (AN,112ss.; 171ss). La pregunta inevitable reza: ¿cuál es la relación entre esas dos tesis? ¿Es una la consecuencia necesaria de la otra? Además, ¿en qué sentido la actual posición implica que, en algún momento, Brentano hubiera pensado lo contrario? Es claro que Brentano ya afirmó no solamente que hay cosas que son y no son

\footnotetext{
23 Véase la síntesis de Franziska Mayer en el prólogo a AN, pp. 1-99.

24 De ahí la expresión "reismo" para designar esta teoría.

25 El «reismo» de Brentano no supone que todo lo real existe sino, únicamente, que si algo existe, entonces es real. El centauro no existe; no dastante, es una casa, pues si existiese sería real.
} 
reales sino además, que nos podíamos representar algo que no es una cosa. Tal era el caso, por ejemplo, del ser y no ser de la cosa. La modificación en la teoría de la verdad como acuerdo exigía correlativamente un ajuste con respecto a lo que podía ser objeto intencional. Con el «Abkehr des Nichtrealen» volvemos a la tesis inicial. Junto con la eliminación de los estados de cosas (Sachverhalten - Sein des Dinges), se va a eliminar también la necesidad de que actos intencionales se dirijan a algo que no es una $\cos ^{26}$.

La teoría de todos y partes tiene, como un caso particular de suma importancia, la relación sustancia-accidente y, por tal motivo, es claro que ha de esperarse que los cambios en la primera conduzcan correlativamente a cambios en la segunda. Así como la tesis de la equivocidad del ser está presupuesta en la primera, también lo está en la segunda. En su "Doctrina de las categorías" (Kategorienlehre) Brentano no está preocupado con qué sustancias hay, sino qué es la sustancia. Siguiendo su principio metódico fundamental, parte de Aristóteles. Sustancia es sinónimo de cosa (Ding, res) y real (Reelles). Lo único que es real es la sustancia y lo único que es sustancia es cosa. Ahora, a partir de 1862, Brentano enseñaba explícitamente que, si la sustancia tiene accidentes y estos están de algún modo contenidos en ella, entonces tienen un ser en la misma, aun cuando, ciertamente, un ser no-real. El accidente depende, en su ser, del ser de la sustancia. Esta concepción se modifica radicalmente a partir de 1904, pasándose a negar todo ser no-real al accidente y, en definitiva, invirtiéndose la relación. El accidente no tiene ahora un ser propio e "irreal" frente a la sustancia. Más aún, no es el accidente parte (irreal) de la sustancia, sino la sustancia parte de un todo (real) que es un todo «accidentado» y que tiene el esencial carácter de ser una cosa. Aquí, una cosa se compone de otra cosa, una cosa tiene como parte real a otra cosa.

Si es cierto que la teoría de la intencionalidad es pensada por Brentano en el marco de una concepción general del ser, es claro que, entonces, cabe esperar que las alteraciones en una, repercutan en la otra. Esto es justamente lo que sucede. Debemos explicitar comparativamente, pues Brentano no lo hace con el suficiente énfasis, cuáles son las consecuencias de esas alteraciones para la teoría de la intencionalidad. Obviamente, si ahora no hay irreales, entonces no puede haber tampoco ni «contenidos» ni el "ser de la cosa". Esto implica una importante reformulación de la teoría de las relaciones dado que para pensar el vínculo intencional ya no son más necesarios dos términos.

\footnotetext{
26 La situación de los «contenidos» (Inhalten) al respecto de esta cuestión es extremadamente más complicada y ha dado lugar a interminables polémicas, debido a las afirmaciones - a primera vista contradictorias - del propio Brentano. Ella merece un estudio detallado que no podemos proporcionar aquí.
} 
Comparando las dos concepciones de intencionalidad de 1874-1891 (PES,PD) y 1904 (K, ANK) tenemos:

A) 1er. concepto de intencionalidad

1. El objeto intencional es inmanente (es un contenido).

2. El objeto intencional no es real "en" la conciencia.

3. El objeto intencional es "en" la conciencia.

4. Lo único real es el acto.

5. Contenidos y ser de la cosa pueden ser objetos intencionales.

6. La relación intencional supone dos términos, ambos existentes, aún cuando uno no sea real.

B) 2do. concepto de intencionalidad

1. El objeto intencional no es inmanente, no es «parte» no-real de un acto real.

2. El objeto intencional no es real "en" la conciencia.

3. El objeto intencional no es una cosa y, en consecuencia, no es en modo alguno.

4. Lo único real es el acto.

5. No hay contenidos ni ser de la cosa, ni ellos pueden ser objetos intencionales.

6. La relación intencional no supone dos elementos (existentes), sino que es posible a partir de un único elemento (real).

La segunda posición de Brentano niega los irreales y, con ellos, los "contenidos". No obstante, ella no implica propiamente un giro a la trascendencia sino todo lo contrario, por lo menos en un sentido negativo, a una inmanencia aún más radical. No es que ahora el acto, en vez de dirigirse a un objeto "en" la conciencia, se dirija a un objeto "fuera" de la conciencia. Es cierto, el acto no contiene ya al objeto como parte no-real de sí. No por eso, sin embargo, menta un algo "trascendente". Para comprender el tipo de "inmanentismo" 27 que Brentano enseña en su última fase, así como las implicaciones del mismo, debemos analizar diferenciadamente el status del objeto intencional en tres casos, a saber, en el caso a) de un objeto «ideal»; b) de un objeto realexistente $y$, finalmente, c) de un objeto real-no existente.

a) La posición final de Brentano de supresión absoluta de la transcendencia elimina toda posibilidad de un reino ideal. En la teoría anterior, la aceptación de contenidos (Inhalten) iba junto con la aceptación del "ser de la cosa" (das Sein des Dinges). Esto implicaba una cierta paradoja; en principio,

27 Podríamos denominar el mismo "inmanentismo negativo" para diferenciarlo de otras formas mas clásicas. Puede cuestionarse si muestra opción es feliz, sobre todo por el hecho de que el propio Brentano, por momentos, parece sentirse más cómodo situándose fuera de la oposición trascendente- inmanente (o "platonismo"-"psicologismo") . 
estas doctrinas parecen ser contradictorias: una apunta en la dirección de un cierto psicologismo, la otra, de un cierto "platonismo". Lo único que las une - y esto de modo puramente negativo - es la tesis de la equivocidad del ser, o sea que no todo lo que es, es real. Lo que contenido y ser de la cosa tenían en común era el hecho de ser sin ser reales. Ahora, por el mismo motivo que se elimina el primero, se elimina el segundo ${ }^{28}$. La lucha contra el ser verdadero en el sentido del ser mental, asumirá la forma de una negación de los contenidos; la lucha contra el ser verdadero, en el sentido de aquello que otorga verdad al juicio, la de una negación del ser de la cosa.

b) Tomemos por caso el hecho de ver un punto rojo. La lectura "trascendentalista" del segundo período diría: en 1874, mi acto se dirige a un rojo «en» la conciencia, mientras que en 1904 al rojo de un objeto «fuera» de ella. Para Brentano, sin embargo, siempre estuvo claro que el rojo no era real ni en, ni fuera de la conciencia. Si el rojo como contenido (Inhalt) ahora desaparece, lo que se coloca en su lugar no es un rojo «trascendente». No existe el rojo, ni en sí, ni en cuanto representado, sino sólo el acto de ver el rojo y éste es real. Antes, como ahora, el objeto intencional no tiene un ser "fuera" de la conciencia. La tesis de la idealidad de las cualidades secundarias no se modifica (K,296-297), como tampoco lo hace la postulación de algún tipo de realidad en sí del mundo físico (K,296-297) ${ }^{29}$. Lo que sufre alteración es que, en la primera teoría de la intencionalidad al rojo le corresponde un ser en la conciencia, o sea, que no sólo el acto de ver el rojo «es», sino también, en algún sentido, el rojo visto; mientras que en la segunda concepción, lo único que resta es el acto de ver el rojo, no habiendo ya lugar para un rojo visto o para un rojo que, de alguna manera sea, aunque no sea real. El rojo visto no sólo no es inmanente, sino que no "es" en modo alguno; lo único que existe existe es el acto de ver el rojo. El rojo jamás está dado de modo directo como algo fuera del acto de visión y al cual este se dirige, sino que está dado sólo de modo oblicuo cuando el hecho de ver el rojo se da de modo directo. Un análisis similar vale para un objeto real cualquiera, por ejemplo, para esta hoja.

c) Finalmente, consideremos el caso de un objeto puramente «imaginario», o «real», mas no existente, por ejemplo un centauro. Lo único que existe en 1904, es el acto de representar el centauro, del cual el centauro no es una parte, ni real, ni ideal. Esto no significa que el centauro, que en 1874 era pensado como objeto inmanente a la conciencia, ahora es pensado de modo

28 El tema del objeto ideal incluye en Brentano el problema de los universales, pero no se reduce a éste. El análisis de Stegnuller en "Hauptströmmungen der gegenwärtigen Philosophie" Suttgart, 7" ed.; pág. 19ss. ) es, en éste punto, unilateral y distorsivo, pues focaliza la doctrina de la negación de los irreales en la de los universales, la afimación de cosas (Dinge), en la del ser individual. En el planteo de Brentano, eso es meramente un aspecto parcial de un contexto mucho más englobante. 
trascendente. Se le niega al centauro un ser "en" la conciencia o un ser en cuanto representado, pero no por ello se le concede un ser "ideal". El centauro no es en modo alguno, ni en la conciencia ni fuera de ella. Lo único que es en sentido propio (o sea, es real), es el acto de representar un centauro y, éste en tanto "cósico" (dinghaft), puede ser representado por nosotros. Ahora, cuando me represento de modo directo un acto intencional, me represento de modo oblicuo su objeto. El centauro sólo puede ser dado de modo oblicuo en el acto de representar de modo directo a alguien que se representa un centauro. Este es el único «ser» del cual él es capaz y es esto lo que quiero decir cuando digo que, en algún sentido, el centauro «es».

\section{La verdad como evidencia}

La lectura inmanentista-negativa propuesta contradice una cierta forma de leer el último Brentano, que hace especial hincapié en un ejemplo célebre dado por el propio filósofo en 1909 (Brentano a Kraus, 12.IX.09 citado en PES,I,XLIX). Cuando prometo casarme con la señorita x, nos dice Brentano, el objeto de la promesa no es mi representación de la señorita $\mathrm{x}$, sino la señorita $\mathrm{x}$ misma. Este ejemplo produce la impresión que la diferencia entre el primer período y el segundo radica en que en el primero el acto se dirige a un objeto «inmanente», mientras que en el segundo a uno «trascendente». En algún momento Brentano habría aceptado entonces el absurdo de que se puede proponer casamiento a una representación (Vorstellung). Sin embargo, pruebas decisivas de que esto no es así - sobre todo por el hecho que ella es totalmente extrínseca e independiente a la evolución del concepto de intencionalidad - y que nuestra lectura "inmanentista-negativa" debe ser mantenida, son las modificaciones que Brentano, sobre el final de su vida, introduce en la teoría de la verdad, pasando de la idea de la verdad como acuerdo a la de la verdad como evidencia (WE,121ss.; AN,179). Ya no hay ahora ni cosas ni el ser de cosas que hagan verdadero al juicio, sino la propia evidencia inmanente al mismo. La evolución de la teoría de la verdad y de la teoría de los irreales, con su postulación y eliminación simultánea de los estados de cosas y contenidos son, pues, plenamente coherentes entre sí. La filosofía de la inmanencia ha llegado a su culminación, afirmando su derecho a no renunciar a toda objetividad y efectuar una fundamentación "sui generis" de la misma. Ella no se resigna ni a ser una variante de relativismo ni tampoco a acep-

29 Subrayemos que no existe en Brentano, en ningún momento, una negación de la realidad fisica trascendente; sea como esta sea pensada y, aun cuando haya diferencias, ciertamente, en el modo de pensarla, su postulación es una constante. 
tar la trascendencia de un reino ideal como única alternativa a éste. Este es el punto central de la polémica del autor de la PES con sus discípulos Husserl y Meinong.

\section{Conclusión}

La doctrina brentaniana de la intencionalidad experimenta una evolución de 1874 a 1904 pudiéndose diferenciar por lo menos dos concepciones excluyentes. Esta evolución acompaña a la de la totalidad del sistema y es consecuencia de ella. El nexo unificador de estos cambios es, en última instancia, la variación del pensamiento del autor con respecto a la tesis aristotélica de la equivocidad del ser. La aceptación de la equivocidad del ser conduce a un modelo de intencionalidad en el cual el objeto intencional es "inmanente" a la conciencia, y aunque no-real, tiene un ser en ella. La negación de la misma tesis conduce a otro modelo intencional que, en vez de ser un giro a a la trascendencia, constituye una radicalización negativa del inmanentismo y, en el cual, se niega al objeto intencional un ser no-real, aceptándose como único ser, el ser real del acto. Reducir la evolución aquí presente como teniendo lugar de la tesis de la inmanencia a la tesis de la transcendencia del objeto intencional ${ }^{30}$, es querer entender la intencionalidad brentaniana en base a parámetros que le son extraños, pues desconoce la importancia de la problemática de la equivocidad del ser, que constituye su contexto sistemático necesario.

\section{Bibliografía}

Von der mannigfachen Bedeutung des Seienden nach Aristoteles. Freiburg, 1862 (VMBSA)

Die Psychologie des Aristoteles, insbesondere seine Lehre vom nous poeitikós. Mainz, $1867(\mathrm{PA})$

Psychologie vom empirischen Standpunkt. Vol. I. Leipzig, 1874 (reimpresión Hamburg,1924) (PES,I); Vol. II. (Ed. Kraus). Hamburg, 1924 (PES,II)

Vom Ursprung sittlicher Erkenntnis. Leipzig, 1889 (reimpresión Hamburg, 1934) (USE)

Deskriptive Psychologie. Aus dem Nachlass herausgegeben und eingeleitet von Roderick M. Chisholm und Wilhelm Baumgartner. Hamburg, 1982 (DP)

Kategorienlehre. Mit Einleitung und Anmerkung herausgegeben von Alfred Kastil. Hamburg,1933 (reimpresión Hamburg, 1985) (K)

30 No menos que negar simplemente toda diferencia entre ambas fases. 
Wahrheit und Evidenz. Erkenntnistheoretische Abhandlungen und Briefe ausgewählt, erläutert und eingeleitet von Oskar Kraus. Hamburg, 1930 (reimpresión, 1974) (WE)

Die Lehre vom richtigen Urteil. Nach den Vorlesungen über Logik mit Benützung anderer Manuskripte aus dem Gebiet der Erkenntnistheorie. Aus dem Nachlasss herausgegeben von Franziska Mayer - Hillebrand. Bern, 1956 (LRU)

Die Abkehr vom Nichtrealen. Mit Einleitung und Anmerkungen von Franziska Mayer Hillebrand. Bern y München, 1966 (AN) 